\title{
Prevalência de Caquexia Neoplásica e Fatores Associados na Internação Domiciliar
}

Prevalence of Cancer Cachexia and its Associated Factors in Home Care

Prevalencia de la Caquexia Neoplástica y sus Factores Asociados en la Atención Domiciliaria

\author{
Patrícia Abrantes Duval' ${ }^{1}$ Rafaela Bülow Bergmann² ; Idrejane Aparecida Viccari do Vale ${ }^{3}$; Catiússa Colling ${ }^{4}$ Évelyn de Sousa Araújo5; Maria Cecília \\ Formoso Assunçãa $0^{6}$
}

\section{Resumo}

Introduçáo: A identificação precoce dos fatores desencadeantes da caquexia oncológica é importante para que a intervenção nutricional auxilie na sua prevenção. Objetivo: Verificar quais são os fatores associados à caquexia em pacientes oncológicos participantes de um programa de internação domiciliar, utilizando conceitos padronizados para a síndrome. Método: Estudo transversal descritivo a partir de dados secundários dos prontuários de todos os pacientes atendidos pelo Serviço de Nutrição, entre fevereiro de 2010 a fevereiro de 2014. A presença de caquexia foi analisada em relação ao sexo, idade, localização do tumor, estadiamento da doença, presença de metástases, tipo de tratamento antineoplásico e sintomas apresentados. Resultados: Foram analisados 276 pacientes, com média de idade de 61,5+13,7 anos, predomínio do sexo masculino (57,3\%), doença avançada (90\%) e presença de metástases $(78,6 \%)$. A prevalência de caquexia foi de $75,3 \%$, estando associada ao estadiamento da doença $(\mathrm{p}=0,001)$, à presença de metástases $(\mathrm{p}=0,002)$ e à localização do tumor $(\mathrm{p}=0,002)$, sendo mais comum entre os portadores de tumores gastrointestinais $(37,3 \%)$. Observou-se associação da caquexia com anorexia $(\mathrm{p}<0,001)$, saciedade precoce $(\mathrm{p}<0,001)$, constipação $(\mathrm{p}=0,02)$, mucosite $(\mathrm{p}=0,02)$, náuseas $(\mathrm{p}=0,03)$, vômitos $(\mathrm{p}=0,01)$, disgeusia $(\mathrm{p}=0,01)$, disosmia $(\mathrm{p}=0,01)$ e dor $(\mathrm{p}=0,01)$. Conclusão: Além dos fatores diretamente relacionados com a doença, tais como: localização do tumor, estadiamento e presença de metástases, diversos sintomas associaram-se à caquexia, e esses merecem atenção diferenciada durante as intervençôes nutricionais. Outros estudos, que avaliem os sintomas associados à caquexia, são necessários para que se possam comparar resultados e estabelecer uma intervenção nutricional.

Palavras-chave: Caquexia; Neoplasias; Serviços de Assistência Domiciliar; Cuidados Paliativos

\footnotetext{
${ }^{1}$ Nutricionista. Mestre em Nutrição e Alimentos pela Universidade Federal de Pelotas (UFPel). Nutricionista do Hospital Escola da UFPel. Pelotas (RS), Brasil. E-mail: patrícia-duvall@hotmail.com.br.

${ }^{2}$ Nutricionista. Mestre em Saúde e Comportamento pela Universidade Católica de Pelotas. Especialista em Nutrição Oncológica pelo programa de Residência Multiprofissional e em Área Profissional da Saúde da UFPel. Pelotas (RS), Brasil. E-mail: bergmann.rafa@gmail.com.

${ }^{3}$ Nutricionista. Especialista em Nutriçăo Oncológica pelo programa de Residência Multiprofissional e em Área Profissional da Saúde da UFPel. Mestranda do Programa de Pós-Graduação em Nutriçāo e Alimentos da UFPel. Pelotas (RS), Brasil. E-mail: idrejanev@gmail.com.

${ }^{4}$ Nutricionista. Especialista em Nutriçấo Oncológica pelo programa de Residência Multiprofissional e em Área Profissional da Saúde da UFPel. Pelotas (RS), Brasil.E-mail: caticolling@hotmail.com.

${ }^{5}$ Nutricionista. Especialista em Nutriçăo Oncológica pelo programa de Residência Multiprofissional e em Área Profissional da Saúde da UFPel. Mestranda do Programa de Pós-Graduaçáo em Nutrição e Alimentos da UFPel. Pelotas (RS), Brasil. E-mail: evelyndsousa@hotmail.com.

${ }^{6}$ Nutricionista. Doutora em Epidemiologia pela UFPel. Pós-doutorado no Instituto Nacional de Salud Pública em Cuernavaca. México. Professora-Associada do Departamento de Nutrição da Faculdade de Nutrição da UFPel. Pelotas (RS), Brasil. E-mail: cecilia.epi@gmail.com.

Endereço para correspondência: Patrícia Abrantes Duval. Pça Cel. Pedro Osório, 158 apto. 72 - Centro. Pelotas (RS), Brasil. CEP: 96015.010.
} 


\section{INTRODUÇÃO}

A caquexia é reconhecida como efeito adverso do câncer, estando associada à redução da função física, à reduzida tolerância ao tratamento antineoplásico e à diminuição da sobrevida ${ }^{1}$. É considerada clinicamente relevante, visto que aumenta a morbidade e mortalidade dos pacientes ${ }^{2}$.

Para auxiliar em estudos, no desenvolvimento de guidelines e no estabelecimento de rotinas clínicas de manejo da caquexia, foi criado em 2011 um consenso internacional para sua definição e classificação. A caquexia associada ao câncer foi então definida como uma síndrome multifatorial, na qual há perda contínua de massa muscular (com perda ou não de massa gorda), que não pode ser totalmente revertida por terapia nutricional convencional, conduzindo ao comprometimento funcional progressivo do organismo. Considerada comum em pacientes com doença avançada, tem um profundo impacto na qualidade de vida, carga de sintomas e sentido da dignidade dos pacientes $^{1,3}$. As manifestaçóes clínicas mais frequentes associadas à síndrome incluem falta de apetite, alteraçôes no paladar, exacerbada perda de peso, anemia, náuseas, astenia, perda de habilidades motoras e físicas, fadiga etc ${ }^{4}$.

Um dos desafios da caquexia no câncer é o controle da perda de peso, relacionada principalmente a alteraçôes metabólicas importantes, à anorexia e redução da ingestáo de calorias, lipídeos e proteínas 5 . A assistência nutricional deve ser vista como parte crucial em intervençôes multimodais voltadas para o tratamento ou prevenção da caquexia, uma vez que sem a alimentação adequada, a estabilização da perda de peso jamais será atingida e a caquexia se manterá em progressão ${ }^{6}$.

Nesse contexto, insere-se o nutricionista no atendimento do Programa de Internação Domiciliar Interdisciplinar (PIDI) Oncológico do Hospital Escola/ Universidade Federal de Pelotas (UFPel), cujo atendimento é prestado exclusivamente pelo Sistema Único de Saúde (SUS). O programa é formado por duas equipes interdisciplinares compostas por médicos, enfermeiros e técnicos de enfermagem, além dos profissionais: nutricionista, assistente social, psicólogo, capelāo, alunos da Residência Multiprofissional em Oncologia/UFPel, alunos de graduação/UFPel e motoristas. Os pacientes são incluídos no programa independentemente da fase de evolução da doença, podendo a admissão estar relacionada a intercorrências inerentes ao tratamento e ao manejo de sintomas? ${ }^{7}$.

Considerando os desafios relacionados à perda de peso progressiva e consequente caquexia do paciente oncológico, torna-se importante a identificação precoce de seus fatores desencadeantes para que uma intervenção nutricional, concomitante a açôes multidisciplinares, possa auxiliar na prevenção e/ou redução da velocidade da perda de peso e no alívio dos sintomas, proporcionando assim uma melhor qualidade de vida ${ }^{8}$. Reconhecendo a importância do tema exposto, o objetivo deste estudo foi verificar quais são os fatores associados à caquexia em pacientes oncológicos participantes de um programa de internação domiciliar, utilizando conceitos padronizados para a síndrome.

\section{MÉTODO}

Estudo transversal descritivo realizado com dados secundários PIDI Oncológico do Hospital Escola/UFPel. Foram incluídos no estudo todos os pacientes atendidos pela equipe da Nutrição no período de fevereiro de 2010 a fevereiro de 2014.

Durante o atendimento nutricional semanal aos pacientes foram coletados dados para avaliaçáo antropométrica, anamnese alimentar e avaliação do estado nutricional por meio da Avaliação Subjetiva Global Produzida pelo Paciente (ASG-PPP) validada no Brasil por Gonzalez et al. ${ }^{9}$. Por via da ASG-PPP, a equipe de nutrição classificou de forma categórica o estado nutricional (ASG A: bem nutrido ou anabólico, ASG B: desnutrição moderada ou suspeita e ASG C: gravemente desnutrido), e determinou uma pontuação numérica, na qual valores iguais ou superiores a quatro indicam risco nutricional e necessidade de intervenção nutricional ${ }^{9,10}$. A classificação categórica e numérica foi coletada para este estudo.

Uma vez que todos os pacientes internados no programa apresentam risco nutricional e necessidade de intervenção, o uso de suplementos nutricionais hipercalóricos e hiperproteicos e receitas de preparaçóes enriquecidas com módulos de proteínas e carboidratos são sempre indicados. Para aquisição dos suplementos, os pacientes são encaminhados à Secretaria Municipal de Saúde e para a Associação de Apoio a Pacientes com Câncer (AAPECAN), que fornece serviços de forma gratuita destinado a pessoas de baixa renda devidamente cadastradas na Instituição.

O critério de diagnóstico de caquexia utilizado neste estudo corresponde ao do Consenso Brasileiro de CaquexialAnorexia em Cuidados Paliativos e do Consenso Internacional para Definição e Classificação de Caquexia no Câncer, que considera perda de peso igual ou superior a $5 \%$ nos últimos seis meses ou de $2 \%$ com um índice de massa corporal (IMC) $<20 \mathrm{Kg} / \mathrm{m}^{2}$, além de redução na ingestão alimentar ${ }^{1,4}$.

A informação sobre alteração da ingestão alimentar foi coletada da ASG-PPP, sendo considerada quando o paciente referia uma diminuição da ingestáo nos 
últimos 30 dias ou alteraçóes relacionadas à consistência e/ou quantidade da dieta tolerada no período da visita domiciliar ${ }^{9}$. A presença de caquexia foi analisada em relação ao sexo, idade, localizaçáo do tumor, estadiamento da doença, presença de metástases, tipo de tratamento antineoplásico e sintomas apresentados pelos pacientes internados no programa.

Entre medicamentos orexígenos prescritos pela equipe, cita-se o uso de corticoide (dexametasona), indicado no manejo da anorexia como agente estimulante do apetite para pacientes portadores de caquexia neoplásica ${ }^{11}$.

Os dados foram digitados no Software Microsoft Excel, exportados e analisados no programa Stata ${ }^{\bullet}$, versão 12.0. Para a descrição das variáveis contínuas, utilizou-se a média com seu respectivo desvio-padrão e, para as variáveis categóricas, o número absoluto e a frequência relativa. O teste de Qui-quadrado foi utilizado para verificar associações entre as variáveis de exposição e desfecho, sendo que todas as análises utilizaram $5 \%$ como nível de significância. O presente estudo foi aprovado pelo Comitê de Ética em Pesquisa da Faculdade de Medicina da UFPel (CAAE 34093914.0.0000.5317).

\section{RESULTADOS}

Foram avaliados pela equipe da Nutrição do PIDI Oncológico 276 pacientes internados no programa no período de fevereiro de 2010 a fevereiro de 2014. A média de idade foi de $61,5+13,7$ anos, sendo predominante o sexo masculino (57,3\%). A maior parte dos pacientes $(90,0 \%)$ apresentou estadiamento avançado da doença e mais de três quartos da amostra $(78,6 \%)$ foram diagnosticados com metástases. Os tumores mais prevalentes foram os gastrointestinais $(36,2 \%)$ e o câncer de pulmão $(14,0 \%)$. O IMC médio correspondeu a 21,5+4,9 $\mathrm{Kgm}^{2}$. A descriçáo completa da amostra encontra-se na Tabela 1.

Os critérios para o diagnóstico de caquexia foram identificados em 271 pacientes, uma vez que faltaram informaçóes nos prontuários sobre a perda de peso ou não foi possível coletar peso e/ou altura de cinco pacientes. Na Figura 1, observam-se a prevalência de caquexia e de seus componentes isolados. A prevalência de caquexia foi de $75,3 \%$; a perda de peso $\geq 5 \%$ ou IMC $<20 \mathrm{Kg} / \mathrm{m}^{2}$ com perda de peso $>2 \%$ foi detectada em mais de $90 \%$ dos pacientes avaliados; enquanto mais de três quartos da amostra apresentaram alteração da ingestão alimentar nos últimos 30 dias ou alteraçóes relacionadas à consistência e/ou quantidade da dieta tolerada no período da visita domiciliar.

A caquexia associou-se à presença de metástases $(\mathrm{p}=0,002)$ e à localização do tumor $(\mathrm{p}=0,002)$, sendo mais comum entre os portadores de tumores gastrointestinais. Além disso, apresentou associação direta com o estadiamento da doença $(\mathrm{p}=0,001)$, sendo que $82,6 \%$ dos pacientes em estadiamento IV apresentavam-se caquéticos. Em relação aos sintomas avaliados por meio da ASG-PPP, somente não foi encontrada associação positiva da presença de caquexia com xerostomia, disfagia e diarreia (Tabela 2). Cabe

Tabela 1. Descrição da amostra. Programa de Internação Domiciliar Interdisciplinar. Pelotas, 2010-2014 ( $n=271)$

\begin{tabular}{|c|c|c|}
\hline & $\mathbf{n}$ & $\%$ \\
\hline \multicolumn{3}{|l|}{ Sexo } \\
\hline Masculino & 156 & 57,6 \\
\hline Feminino & 115 & 42,4 \\
\hline \multicolumn{3}{|l|}{ Tratamento antineoplásico } \\
\hline Quimioterapia & 216 & 79,7 \\
\hline Radioterapia & 121 & 44,7 \\
\hline Cirurgia & 135 & 49,8 \\
\hline \multicolumn{3}{|l|}{ Estadiamento } \\
\hline I & 1 & 0,4 \\
\hline II & 26 & 9,6 \\
\hline III & 72 & 26,6 \\
\hline IV & 172 & 63,5 \\
\hline Metástase & 213 & 78,6 \\
\hline \multicolumn{3}{|l|}{ Localização do tumor } \\
\hline Pulmão & 38 & 14,0 \\
\hline Gastrintestinais & 98 & 36,1 \\
\hline Ginecológicos & 35 & 12,9 \\
\hline Cabeça/pescoço & 26 & 09,6 \\
\hline Outros & 74 & 27,3 \\
\hline \multicolumn{3}{|l|}{ Sintomas } \\
\hline Anorexia & 127 & 46,9 \\
\hline Dor & 122 & 45,0 \\
\hline Saciedade & 96 & 35,4 \\
\hline Xerostomia & 88 & 32,5 \\
\hline Náuseas & 86 & 31,7 \\
\hline Constipação & 69 & 25,5 \\
\hline Disfagia & 66 & 24,4 \\
\hline Vômitos & 59 & 21,8 \\
\hline Disgeusia & 48 & 17,7 \\
\hline Disosmia & 43 & 15,9 \\
\hline Mucosite & 29 & 10,7 \\
\hline Diarreia & 25 & 09,2 \\
\hline Uso de opioide & 147 & 54,2 \\
\hline \multicolumn{3}{|l|}{ Estado nutricional } \\
\hline A (bem nutrido) & 03 & 01,1 \\
\hline B (desnutrição moderada) & 119 & 43,9 \\
\hline $\mathrm{C}$ (gravemente desnutrido) & 149 & 55,0 \\
\hline \multicolumn{3}{|l|}{ IMC } \\
\hline Déficit de peso & 71 & 28,5 \\
\hline Peso adequado & 120 & 48,2 \\
\hline Sobrepeso & 45 & 18,1 \\
\hline Obesidade & 13 & 05,2 \\
\hline
\end{tabular}




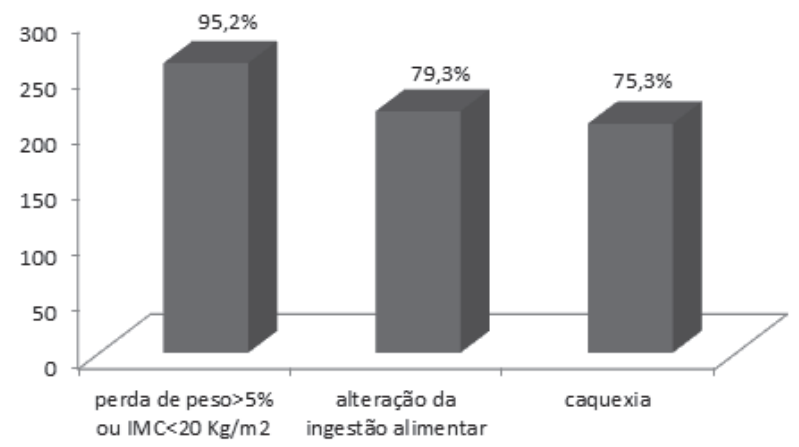

Figura 1. Prevalência de caquexia e suas características isoladas. Programa de Internação Domiciliar Interdisciplinar. Pelotas, 2010 $2014(n=271)$

salientar que os pacientes podem ter sido submetidos a um ou mais tratamentos antineoplásicos, assim como o relato da presença de um ou mais sintomas. Dessa forma, o somatório da Tabela 2 é maior que o número total de pacientes avaliados neste estudo.

Entre os pacientes classificados com caquexia, 134 $(65,7 \%)$ utilizaram o corticoide dexametasona e, a terapia nutricional enteral foi instituída em dez pacientes (4,9\%), sendo sete por via de gastrostomias/jejunostomias e três por sonda nasoenteral.

\section{DISCUSSÃO}

A prevalência de caquexia foi bastante elevada entre os pacientes internados no PIDI oncológico. Fatores inerentes à progressão e gravidade da doença, tais como: presença de metástases, localizaçáo tumoral e estadiamento da doença, foram fortemente associados à síndrome.

Estudo realizado no mesmo programa, entre 2006 e 2008, verificou prevalência de caquexia em aproximadamente $50 \%$ dos pacientes no momento da internação, utilizando o critério diagnóstico recomendado pela Organización Panamericana de la Salude International Association for Hospice and Palliative Care ${ }^{12,13}$.

Sintomas que atingem diretamente a ingestáo alimentar, tais como: anorexia, náuseas, vômitos, saciedade, mucosite, disgeusia, disosmia e perda de peso, também foram significativamente associados à caquexia e merecem atenção diferenciada durante as intervençóes nutricionais. Alguns desses sintomas também estiveram presentes nos 4.822 pacientes hospitalizados com câncer e avaliados com a ASG-PPP no inquérito nutricional multicêntrico realizado pelo Instituto Nacional de Câncer José Alencar Gomes da Silva (INCA). A anorexia, náusea e a disosmia estavam entre os principais sintomas gastrointestinais apresentados tanto para adultos quanto para idosos, tendo uma alta prevalência de perda de peso ${ }^{14}$.
Tabela 2. Caquexia e fatores associados. Programa de Internação Domiciliar Interdisciplinar. Pelotas, 2010-2014 (n=204)

\begin{tabular}{|c|c|c|c|}
\hline Caquexia & $\mathbf{N}$ & $\%$ & Valor $p^{\prime}$ \\
\hline Sexo & & & 0,89 \\
\hline Masculino & 118 & 57,8 & \\
\hline Feminino & 86 & 42,2 & \\
\hline \multicolumn{4}{|l|}{$\begin{array}{l}\text { Tratamento } \\
\text { antineoplásico }\end{array}$} \\
\hline Quimioterapia & 163 & 79,9 & 0,86 \\
\hline Radioterapia & 87 & 42,6 & 0,26 \\
\hline Cirurgia & 100 & 49,0 & 0,68 \\
\hline Estadiamento & & & 0,001 \\
\hline I & 01 & 0,5 & \\
\hline II & 19 & 9,3 & \\
\hline III & 42 & 20,6 & \\
\hline IV & 142 & 69,6 & \\
\hline Metástases & 170 & 83,3 & 0,002 \\
\hline Localização do tumor & & & 0,02 \\
\hline Pulmão & 30 & 14,7 & \\
\hline Gastrintestinais & 76 & 37,3 & \\
\hline Ginecológicos & 28 & 13,7 & \\
\hline Cabeça/pescoço & 12 & 5,9 & \\
\hline Outros & 58 & 28,4 & \\
\hline \multicolumn{4}{|l|}{ Sintomas } \\
\hline Anorexia & 114 & 55,8 & $<0,001$ \\
\hline Náuseas & 72 & 35,3 & 0,03 \\
\hline Vômitos & 52 & 25,5 & 0,01 \\
\hline Constipação & 59 & 28,9 & 0,02 \\
\hline Diarreia & 22 & 10,8 & 0,15 \\
\hline Mucosite & 27 & 13,2 & 0,02 \\
\hline Xerostomia & 71 & 34,8 & 0,18 \\
\hline Disgeusia & 43 & 21,1 & 0,01 \\
\hline Disosmia & 39 & 19,1 & 0,01 \\
\hline Disfagia & 46 & 22,5 & 0,25 \\
\hline Saciedade & 87 & 42,6 & $<0,001$ \\
\hline Dor & 102 & 50,0 & 0,01 \\
\hline Estado nutricional & & & 0,24 \\
\hline A & 01 & 0,5 & \\
\hline B & 91 & 44,6 & \\
\hline C & 112 & 54,9 & \\
\hline $\mathrm{IMC}^{\|}$ & & & 0,91 \\
\hline Déficit de peso & 51 & 27,3 & \\
\hline Peso adequado & 91 & 48,7 & \\
\hline Sobrepeso & 35 & 18,7 & \\
\hline Obesidade & 10 & 5,3 & \\
\hline
\end{tabular}

I Teste Exato de Fischer.

II $\mathrm{n}=187$.

O estado nutricional avaliado por meio do IMC e da ASG-PPP não demostraram associação com a caquexia, comprovando que essas não são ferramentas sensíveis para detectar a depleção nutricional causada pela síndrome. Xerostomia, disfagia e diarreia também não foram relacionadas à presença de caquexia. 
De acordo com o Consenso Brasileiro de Caquexial Anorexia, mais de três quartos dos portadores de doença avançada apresentam a caquexia induzida pelo câncer ${ }^{4}$. Além disso, segundo Gullet et al. ${ }^{15}$, a síndrome é mais comum na doença avançada entre os pacientes com tumores do trato gastrointestinal, pâncreas, tórax e cabeça e pescoço, órgãos responsáveis pela nutrição como um todo (ingestão, absorção e utilização de nutrientes). $\mathrm{Na}$ amostra de pacientes do PIDI, houve predomínio de doença avançada (estadiamentos III e IV) em aproximadamente $90 \%$ dos indivíduos avaliados. Além disso, metástases e tumores primários gastrointestinais e de pulmáo acometeram mais da metade dos pacientes estudados. Sabe-se que, somados, os tumores de trato gastrointestinal e pulmão podem determinar a presença de caquexia entre $60 \%$ e $80 \%$ dos pacientes já no momento do diagnóstico ${ }^{16}$. Juntos, todos esses fatores podem ter influenciado a alta prevalência de caquexia entre os pacientes domiciliares internados no programa.

Um problema dos estudos realizados até hoje sobre a relação dos fatores associados à caquexia é relatado por Blum et al. ${ }^{5}$. Em razão da heterogeneidade dos conceitos utilizados para caquexia, é difícil determinar com consistência os sintomas associados à síndrome e as intervençōes que efetivamente contribuem para amenizar o quadro 5 . Alguns sintomas gastrointestinais como: náuseas, vômitos, anorexia, mucosite e disgeusia contribuem para o desenvolvimento da caquexia ${ }^{17}$. No presente estudo, a maior parte desses fatores que influenciam a perda de peso, como anorexia, esteve associada à caquexia. Torna-se importante que outros estudos, utilizando a mesma definição para caquexia, sejam realizados a fim de permitir futuras comparações.

No que se refere à perda ponderal, encontrou-se uma prevalência superior a $90 \%$ da amostra estudada. Alguns fatores etiológicos da perda de peso são bem conhecidos e incluem o estadiamento, a localização tumoral e a anorexia ${ }^{18,19}$, sendo essa última encontrada em quase metade dos pacientes avaliados. Mais do que qualquer outro sintoma, a anorexia é vista como parte dos primeiros sinais clínicos e metabólicos que precedem à caquexia, podendo induzir perda de peso substancial e involuntária ${ }^{18}$.

Além da sintomatologia associada diretamente à redução da ingestão, o próprio avanço da doença provoca a liberação de citocinas e outros mediadores metabólicos que suprimem o apetite e causam saciedade precoce, alteração da ingestão e perda de peso ${ }^{20}$. Considerando que a alteraçáo de ingestão foi o sintoma mais prevalente entre os pacientes do PIDI e esteve fortemente associada à caquexia $(\mathrm{p}<0,001)$, é relevante atentar para o fato de que esse pode ser um dos sintomas mais desafiadores no controle da perda de peso e manifestação da caquexia.
A utilização de nutrientes específicos associados a estimulantes do apetite pode gerar um ambiente positivo para prevenir a perda ponderal. Segundo Fearon et al. ${ }^{1}$, pacientes caquéticos devem receber aconselhamento para alimentaçáo adequada, estimuladores orais de apetite e suplementos conforme a necessidade, além de serem tratados em relaçáo à inflamação sistêmica. Apesar de essa conduta estar cada vez mais próxima de ser inserida nas rotinas de atendimento nutricional, ainda existe uma carência muito grande de informaçóes que permitam concretizar padrōes de condutas. Por isso, os detalhes precisos desse tipo de intervenção devem ser explorados em ensaios clínicos randomizados para que sejam aplicados de forma segura e efetiva na prática clínica.

Considera-se que esse achado seja um ponto de partida para orientação das condutas nutricionais e multiprofissionais, a fim de proporcionar alívio de sintomas, reduzir a progressão da perda de peso, e melhorar a qualidade de vida. Apesar de a literatura ser controversa em relação aos benefícios da intervenção dietética em pacientes caquéticos ${ }^{15}$, o acompanhamento nutricional, visando à redução e ao controle dos sintomas, deve ser sempre indicado, uma vez que pode contribuir para amenizar a anorexia e evitar a perda de peso ${ }^{21}$. Independente de qualquer intervenção, sem a alimentação adequada, a estabilização da perda de peso não será alcançada ${ }^{6}$.

Um estudo sobre manejo de sintomas que induzem a caquexia em pacientes em cuidados paliativos também mostrou que uma avaliaçáo padronizada dos sintomas associados à caquexia pode contribuir significativamente no manejo e no impacto desses sobre o estado nutricional por meio de medidas simples e que podem ser aplicadas por toda a equipe multidisciplinar ${ }^{22}$. $\mathrm{O}$ mesmo estudo aponta que é possível que muitos problemas do paciente, percebidos pelo profissional de saúde, podem ser diferentes daqueles realmente vivenciados pelo indivíduo; e, por isso, o uso de uma ferramenta padronizada para avaliação dos sintomas pode ser um passo importante no manejo da sintomatologia que induz a caquexia.

\section{CONCLUSÃO}

A prevalência de caquexia detectada entre os pacientes estudados foi elevada, uma vez que a maioria encontrava-se em cuidados paliativos, com doença avançada, importante perda de peso e redução da ingestão alimentar.

Neste estudo, observou-se que, além dos fatores diretamente relacionados com a doença, tais como: localização do tumor, estadiamento da doença e presença de metástases, diversos sintomas associaram-se à caquexia, e esses merecem atenção diferenciada durante as intervenções nutricionais. 
Existem poucos estudos realizados com pacientes em cuidados paliativos utilizando o conceito de caquexia de acordo com o do Consenso Brasileiro de CaquexialAnorexia em Cuidados Paliativos e do Consenso Internacional para Definição e Classificação de Caquexia no Câncer. Sendo assim, outros estudos, que avaliem os sintomas associados à síndrome diagnosticada por esses conceitos, são necessários para que se possam comparar resultados e estabelecer uma intervençấo nutricional que auxilie na prevenção e/ou redução da velocidade da perda de peso e no alívio dos sintomas.

\section{CONTRIBUIÇÕES}

Ambas as autoras contribuíram na concepção e planejamento do projeto de pesquisa, na obtençáo, análise e interpretação dos dados, e na redação final e revisão crítica do manuscrito.

Declaraçáo de Conflito de Interesses: Nada a Declarar.

\section{REFERÊNCIAS}

1. Fearon K, Strasser F, Anker SD, Bosaeus I, Bruera E, Fainsinger RL, et al. Definition and classification of cancer cachexia: an international consensus. Lancet Oncol. 2011 May;12(5):489-95.

2. Muscaritoli M, Anker SD, Argilés J, Aversa Z, Bauer JM, Biolo G, et al. Consensus definition of sarcopenia, cachexia and pre-cachexia: Joint document elaborated by Special Interest Groups (SIG) "cachexia-anorexia in chronic wasting diseases" and "nutrition in geriatrics". Clin Nutr. 2010 Apr;29(2):154-9.

3. Del Fabbro E, Inui A, Strasser F. Cancer cachexia. London: Springer Healthcare; 2012.

4. Associação Brasileira de Cuidados Paliativos. Consenso Brasileiro De Caquexia e Anorexia em Cuidados Paliativos. Rev Bras Cuidados Paliativos. 2011;3(3 Supl $1): 3-42$.

5. Blum D, Omlin A, Baracos VE, Solheim TS, Tan $\mathrm{BH}$, Stone P, et al.; European Palliative Care Research Collaborative. Cancer cachexia: a systematic literature review of items and domains associated with involuntary weight loss in cancer. Crit Rev Oncol Hematol. 2011 Oct;80(1):114-44.

6. Balstad TR, Solheim TS, Strasser F, Kaasa S, Bye A. Dietary Treatment of Weight loss in patients with advanced cancer and cachexia: a systematic literature review. Crit Rev Oncol Hematol. 2014 Aug;91(2):210-21.

7. Arrieira ICO, Thofehrn MB, Fripp JC, Duval P, Valadão M, Amestoy SC. Programa de internação domiciliar interdisciplinar oncológico: metodologia de trabalho. Ciênc. cuid. Saúde. 2009;8(Supl):104-9.

8. Morley JE. Calories and cachexia. Curr Opin Clin Nutr Metab Care. 2009 Nov;12(6):607-10.

9. Gonzalez MC, Borges LR, Silveira DH, Assunção MCF, Orlandi SP. Validação da versão em português da avaliação subjetiva global produzida pelo paciente. Rev Bras Nutr Clin. 2010;25(2):102-8.

10. Waitzberg DL. Dieta, nutrição e câncer. São Paulo: Atheneu; 2006.

11. Carvalho RT, Parsons HÁ, organizadores. Manual de Cuidados Paliativos ANCP. 2a edição ampliada e atualizada. São Paulo: ANCP; 2012.

12. Duval AD, Vargas BL, Fripp JC, Arrieira ICO, Lazzeri B, Destri K. Caquexia em pacientes oncológicos internados em um programa de internação domiciliar interdisciplinar. Rev Bras Cancerol. 2010;56(2):207-12.

13. Nervi F. Anorexia y caquexia. In: Bruera E, Lima L, editores. Cuidados Paliativos: Guias para el Manejo Clínico [Internet]. [Washington]: OPAS; [data desconhecida] [acesso em $10 \mathrm{Fev}$ 2008]. Disponível em: http://www.paho.org/spanish/ad/dpc/nc/palliativecare.pdf.

14. Instituto Nacional de Câncer José Alencar Gomes da Silva. Inquérito brasileiro de nutrição oncológica. Rio de Janeiro: Inca; 2013.

15. Gullett NP, Mazurak VC, Hebbar G, Ziegler TR. Nutritional interventions for cancer-induced cachexia. Curr Probl Cancer. 2011 Mar-Apr;35(2):58-90.

16. Springer J, Von Haehling S, Anker SD. The need for a standardized definition for cachexia in chronic illness. Nat Clin Pract Endocrinol Metab. 2006 Aug;2(8):416-7.

17. Holmes $S$. Nutrition in the care of patients with cancer cachexia. Br J Community Nurs. 2011 Jul;16(7):314, 316, 318 passim.

18. Argilés JM, Alvarez B, López-Soriano FJ. The metabolic basis of câncer cachexia. Med Res Rev. 1997 Sep;17(5):477-98.

19. Fearon KC, Moses AG. Cancer Cachexia. Int J Cardiol. 2002;85(1):73-81.

20. Hopkinson JB, Wright DN, Foster C. Management of weight loss and anorexia. Ann Oncol. 2008 Sep; 19 Suppl 7:vii289-93.

21. Nitenberg G, Raynard B. Nutritional support of the câncer patient: issues and dilemmas. Crit Rev Oncol Hematol. 2000 Jun;34(3):137-68.

22. Andrew IM, Waterfield K, Hildreth AJ, Kirkpatrick G, Hawkins C. Quantifying the impact of standardized assessment and symptom management tools on symptoms associated with cancer-induced anorexia cachexia syndrome. Palliat Med. 2009 Dec;23(8):680-8. 


\section{Abstract}

Introduction: Early identification of the factors triggering cancer cachexia is important for the nutritional intervention to assist in its prevention. Objective: To determine which factors are associated with cachexia in cancer patients participating in a home care program, using standard concepts for the syndrome. Method: A descriptive cross-sectional study using secondary data from medical records of all patients attended by the Nutrition Service staff from February 2010 to February 2014. The presence of cachexia was analyzed in relation to gender, age, tumor location, stage of disease, presence of metastases, type of anti-cancer treatment and symptoms presented. Results: There were 276 patients, mean age $61.5 \pm 13.7$ years, predominance of male gender $(57.3 \%)$, advanced disease $(90 \%)$ and metastasis $(78.6 \%)$. The prevalence of cachexia was $75.3 \%$, and it was associated with disease stage $(\mathrm{p}=0.001)$, the presence of metastases $(\mathrm{p}=0.002)$ and tumor location $(\mathrm{p}=0.002)$, and it was more common among patients with gastrointestinal tumors (37.3\%). There was an association between cachexia and anorexia $(\mathrm{p}<0.001)$, early satiety $(\mathrm{p}<0.001)$, constipation $(\mathrm{p}=0.02)$, mucositis $(\mathrm{p}=0.02)$, nausea $(\mathrm{p}=0.03)$, vomiting $(\mathrm{p}=0.01)$, dysgeusia $(\mathrm{p}=0.01)$, dysosmia $(\mathrm{p}=0.01)$ and pain $(\mathrm{p}=0.01)$. Conclusion: In addition to the factors directly related to the disease, such as tumor location, stage and metastasis, several symptoms were associated with cachexia, and they deserve special attention during nutritional interventions. Other studies assessing symptoms associated with cachexia are needed so that they can compare results and establish a nutritional intervention.

Key words: Cachexia; Neoplasms; Home Care Services; Palliative Care

\section{Resumen}

Introducción: La identificación temprana de los factores desencadenantes de la caquexia oncológica es importante para intervención nutricional para para ayudar en su prevención. Objetivo: Determinar qué factores se asocian con la caquexia en pacientes con cáncer que participaban en un programa de atención a domicilio, utilizando conceptos estándar para el síndrome. Método: Estudio descriptivo transversal utilizando datos secundarios de las historias clínicas de todos los pacientes tratados por el Servicio de la Nutrición febrero del 2010 hasta febrero del 2014. La caquexia se analizó en relación con el sexo, edad, localización del tumor, estadio de la enfermedad, presencia de metástasis, tipo

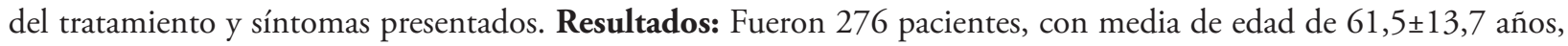
predominio del sexo masculino $(57,3 \%)$, enfermedad avanzada (90\%) y metástasis $(78,6 \%)$. La prevalencia de caquexia fue del $75,3 \%$, siendo asociada con estadio del enfermedad ( $\mathrm{p}=0,001)$, presencia de metástasis $(\mathrm{p}=0,002)$ y estadio del tumor $(\mathrm{p}=0,002)$, y fue más común entre pacientes con tumores gastrointestinales $(37,3 \%)$. Se observó anorexia asociada con la caquexia $(\mathrm{p}<0,001)$, saciedad temprana $(\mathrm{p}<0,001)$, estreñimiento $(\mathrm{p}=0,02)$, mucositis $(\mathrm{p}=0,02)$, náuseas $(\mathrm{p}=0,03)$, vómitos $(\mathrm{p}=0,01)$, disgeusia $(\mathrm{p}=0,01)$, disosmia $(\mathrm{p}=0,01)$ y el dolor $(\mathrm{p}=0,01)$. Conclusión: Además de los factores directamente relacionados con la enfermedad, tales como estadio y metástasis, varios síntomas se asociaron con caquexia, y merecen una atención especial durante las intervenciones nutricionales. Se necesitan otros estudios de evaluación de los síntomas asociados con la caquexia, para que se puedan comparar los resultados y establecer una intervención nutricional.

Palabras clave: Caquexia; Neoplasias; Servicios de Atención de Salud a Domicilio; Cuidados Paliativos 